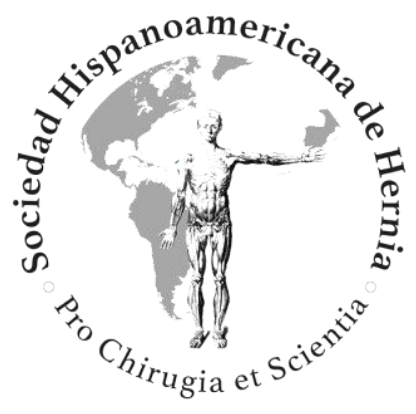

Perforación por hueso de pollo en asa intestinal incarcerada de hernia incisional

\title{
Perforation by chicken bone in incarcerated intestinal loop of incisional hernia
}




\section{346}

Perforación por hueso de pollo en asa intestinal incarcerada de hernia incisional

Perforation by chicken bone in incarcerated intestinal loop of incisional hernia

Manuel Antonio Grez Ibáñez ${ }^{1}$, Antonio Gambardella Flores², Amaranda Silva Torres $^{3}$

Servicios de ${ }^{1}$ Cirugía y de ${ }^{2}$ Urgencias. Hospital San Juan de Dios de Curicó (Chile). ${ }^{3}$ Facultad de Medicina Universidad de Talca (Chile)

Recibido: 05-06-2020

Aceptado: 10-06-2020

Autor para correspondencia: Manuel Antonio Grez Ibáñez. Hospital San Juan de Dios de Curicó. Av. San Martín, Curicó, Maule (Chile)

Correo electrónico: magrezster@gmail.com

DOI: $10.20960 /$ rhh.00346

Conflicto de interés: los autores declaran no tener conflicto de intereses.

\section{RESUMEN}

Introducción: La ingesta de cuerpos extraños suele ser un motivo de consulta frecuente. En un $80 \%$ de los casos pasan desapercibidos y sin dar molestias a través del tubo digestivo y pueden tener múltiples tamaños y formas, pero los delgados, duros y afilados como son las espinas de pescado, trozos de huesos de pollo o limpiadientes, tienden a detenerse en localizaciones más estrechas del tracto digestivo. Al ser puntos de impacto, 
pueden provocar una perforación intestinal y debutar con una peritonitis aguda que requerirá un diagnóstico y una cirugía precoz.

Caso clínico: Presentamos el caso clínico de una mujer de 56 años, portadora de una abdominoplastia y hernioplastia con malla protésica por hernia incisional poscesárea 12 años antes y recidivada a los 3 años, que consultó en urgencia por perforación intestinal en asa herniada y acodada por debajo de la malla en segmento de íleon de hernia incisional por hueso de pollo. Se diagnostica precozmente por tomografía axial computarizada abdominal con resección intestinal de urgencia y se difiere la hernioplastia definitiva por peritonitis localizada.

Discusión: La presentación y diagnóstico de esta paciente constituyó un verdadero desafío diagnóstico por carecer del dato anamnésico de la ingesta de un cuerpo extraño, con sintomatología sugerente de hernia incisional atascada. El diagnóstico por tomografía ayudó en la certeza diagnóstica y en su cirugía precoz. El acodamiento del asa herniada sirvió como punto de impacto para su perforación y la peritonitis local obligó a diferir la colocación de malla.

Conclusión: Debemos recordar la posibilidad de un cuerpo extraño como causa de obstrucción y abdomen agudo por perforación intestinal en cierto tipo de pacientes (ancianos, mala dentadura, etc.), para poder evitar demoras innecesarias que puedan retrasar la cirugía y elevar la morbilidad. Un cuerpo extraño puede complicar la evolución de una recidiva ya conocida.

Palabras clave: Perforación intestinal, ingestión de cuerpo extraño, hueso de pollo, hernia incisional.

\section{ABSTRACT}

Introduction: Ingestion of foreign bodies is a frequent reason for consultation. In $80 \%$ of the cases these go unnoticed and without discomfort through the digestive tract. They may have multiple sizes and shapes, but 
the thin, hard and sharp ones such as fish bones, chicken bones or toothpicks, tend to stop at narrower locations in the digestive tract. Since they are points of impact, they can cause an intestinal perforation and debut with acute peritonitis that will require early diagnosis and surgery.

Case report: We present a clinical case of a 56-year-old female patient, who had abdominoplasty and hernioplasty with a prosthetic mesh for incisional hernia post-cesarean 12 years ago with a recurrence 3 years ago. Consulted in the emergency department for intestinal perforation in herniated loop and bent below the mesh in ileum segment of incisional hernia due to a foreign body (chicken bone). She was diagnosed early by abdominal computerized tomography with emergency intestinal resection, and the hernioplasty was deferred because of a localized peritonitis.

Discussion: Presentation and diagnosis of this patient constituted a real diagnostic challenge due to the lack of anamnestic data on foreign body intake, with symptoms suggestive of a stuck incisional hernia. Diagnosis by tomography helped in the diagnostic certainty and the early surgery. Kinking of herniated loop served as an impact point for the perforation and local peritonitis forced the mesh placement to be deferred.

Conclusion: We must remember the possibility of a foreign body as a cause of obstruction and an acute abdomen due to intestinal perforation in certain types of patients (elderly, bad teeth, etc.) in order to avoid unnecessary waiting periods that may delay surgery and increase morbidity. A foreign body can complicate the evolution of a known recurrence.

Keywords: Intestinal perforation, ingestion of strange body, chicken bone, incisional hernia.

\section{INTRODUCCIÓN}

La ingestión de cuerpos extraños (CE) es un motivo de consulta frecuente en los servicios de urgencia. En un $80-90 \%$ de los casos se ingiere de forma desapercibida y gran parte de ellos atraviesa el tubo digestivo de forma 
asintomática y espontánea ${ }^{1,2}$. El tipo de CE más común varía según la edad: en los niños son más frecuentes los CE inorgánicos como monedas, botones, pilas, objetos de plástico, etc. y en adultos (especialmente en ancianos), son Ios CE orgánicos como los bolos alimentarios mal masticados, trozos de huesos, huesos de pescado ${ }^{1,3}$, tallos vegetales. Así fue en el caso reportado por Rodríguez-Hermosa ${ }^{3}$ de una anciana operada de peritonitis aguda donde se encontró una perforación intestinal por una ramita de tomillo, así como palillos y prótesis dentales ${ }^{3,13}$. Un grupo aparte lo constituyen los que ingieren CE de forma intencional, como son los contrabandistas (mulas) de drogas ilegales, joyas y otros objetos de valor para evadir la detención por parte de los cuerpos seguridad ${ }^{2}$.

Para los CE que provocan síntomas y quedan retenidos en el tracto superior se puede requerir una extracción endoscópica, tal y como lo publica Ramos ${ }^{6}$ con una extracción de hueso de pollo impactado en la pared gástrica, requiriéndose cirugía en el $1 \%$ por perforación intestinal. Esta es la complicación más temida, y se expone en otro caso reportado por RodríguezHermosa ${ }^{6}$ de una paciente con abdomen agudo por perforación intestinal de espina de pescado que falleció a pesar de haber sido operada con resección intestinal y tratamiento en $\mathrm{UCl}$.

Hay poblaciones con mayor riesgo de perforación intestinal por CE como son los enfermos mentales, alcohólicos, prisioneros, carpinteros, modistas y las personas con prótesis dentales, ya que disminuyen el nivel de sensibilidad oral$^{2}$. Hay ciertas localizaciones que por su estrechez son puntos de impacto como: el píloro, ángulo de Treitz, válvula ileocecal ${ }^{1}$ o la unión rectosigmoídea $3,7,13,14$, que además pueden ser favorecidas por zonas de adherencias, áreas con procesos diverticulares, anastomosis quirúrgicas o en un divertículo de Meckel, como el caso reportado por $\mathrm{Chan}^{9}$, donde se encontró un hueso en un joven al realizarle una laparoscopia por apendicitis aguda.

EI CE más común en las perforaciones intestinales es la espina de pescado ${ }^{1}$ con una obvia asociación directa con la frecuencia de consumo de pescado 
en la dieta local, siendo esta mayor en algunas razas orientales ${ }^{3,6,10,11}$. Es precisamente este tipo de perforación el más antiguo encontrado en la literatura médica cuando Nonat ${ }^{12}$, médico parisino, lo publicó en el año 1841 (fig. 1).

Las perforaciones intestinales pueden tener diferentes presentaciones según el sitio anatómico de la lesión, el compromiso de los órganos adyacentes y el tiempo de evolución de los síntomas. Generalmente debutan con diagnósticos de úlcera perforada, peritonitis aguda (localizada o generalizada), o como una apendicitis aguda ${ }^{13}$. Piñero ${ }^{14}$, hace 40 años, reportaba en un estudio retrospectivo de 18 años que en su hospital encontraron 21 pacientes que aunque plantearan los mismos diagnósticos que en anteriores casos por ingesta de cuerpo extraño, solo pudieron llegar a un diagnóstico etiológico en el mismo acto quirúrgico. Hay que tener en cuenta que esto era en un tiempo en que solo se disponía de placas radiográficas como único método diagnóstico imagenológico y en las que solo en un $20 \%$ mostraron un neumoperitoneo. El cuadro inicial es inespecífico: la mayoría de los pacientes progresan hacia la peritonitis localizada y el diagnóstico preoperatorio se confunde con apendicitis o diverticulitis aguda, dependiendo del lugar de la sintomatología ${ }^{14}$.

Aunque el tiempo de paso del CE por el tubo digestivo se calcula en una semana, se han descrito casos de meses y hasta años desde el momento de la ingestión hasta el inicio de los síntomas. Hace unas décadas se solían usar las placas radiográficas simples como método diagnóstico ${ }^{14}$, y tenían mal rendimiento para visualizar CE vegetales o espinas de pescado, así como los huesos de pollo. Magline ${ }^{15}$ reportó en el año 1979 cuatro casos de perforación por hueso de pollo y en dos este método fue incapaz de mostrarlos. Vasapollo ${ }^{16}$ reportó un caso el año 1997, en el que tampoco se pudo llegar al diagnóstico, y es actualmente el TAC la prueba considerada de elección, porque permite identificar la presencia, el sitio y la causa de la perforación intestinal, con un $100 \%$ de capacidad para demostrar aire libre en todos los casos ${ }^{8}$. 
La perforación intestinal es una urgencia quirúrgica que requiere un diagnóstico rápido ${ }^{8,9}$, así como un tratamiento quirúrgico de urgencia y tratamiento antibiótico, tal como insiste el cirujano español RodríguezHermosa ${ }^{17,18}$ en todos sus reportes y a quien este tema parece haberle interesado especialmente ${ }^{5,8}$. Acosta ${ }^{3}$ y Soria ${ }^{4}$ reportan que su resolución también puede ser planteada por vía laparoscópica. La pronta resolución de la causa desencadenante disminuye la posibilidad de que se desarrolle un síndrome de respuesta inflamatoria sistémica, sepsis o fallo orgánico múltiple. Las medidas de soporte hídrico, antibiótico y vasopresor, si se requiere, acompañadas de una temprana intervención quirúrgica, control y seguimiento posoperatorio, son vitales en este tipo de pacientes ${ }^{10}$.

\section{CASO CLÍNICO}

Mujer de 56 años, obesa mórbida con un IMC de $40 \mathrm{~kg} / \mathrm{cm}^{2}$, con dentadura parcial y uso de prótesis dental en mal estado, con antecedente quirúrgico de herniorrafia de pared abdominal anterior media infraumbilical hace 12 años y con colocación de malla protésica supraaponeurótica con abdominoplastia (sin neoimplante umbilical), por una hernia incisional poscesárea. Consulta en nuestro servicio de Urgencia por dolor agudo e intenso de 24 horas de evolución en región infraumbilical derecha, en relación a un aumento de volumen del subcelular aparecida hacía 3 años, sugerente de una recidiva herniaria ahora complicada, pero sin signos sépticos o de obstrucción intestinal y con la paciente estable, afebril e hidratada. En el examen físico abdominal se encuentra un panículo adiposo grueso, con ausencia de ombligo y extensa cicatriz transversa en parte inferior por abdominoplastia antigua con un aumento del volumen herniario por debajo del grueso panículo adiposo, de $6 \times 4 \mathrm{~cm}$ aproximadamente en región medial de fosa iliaca derecha, muy dolorosa a la palpación pero sin signos de irritación peritoneal. En sus exámenes preoperatorios destaca una leucocitosis de $15.000 \mathrm{~GB}$ por $\mathrm{ml}$ y una PCR en 3.5; el resto de exámenes en límites normales. 
Se realizó TAC de abdomen y pelvis con contraste que mostró un orificio herniario de $5 \times 3 \mathrm{~cm}$ en línea media infraumbilical con asas de intestino delgado herniadas sobre región supraaponeurótica derecha inferior, con una de ellas perforada por cuerpo extraño de $40 \times 3 \mathrm{~mm}$ con discreto neumoperitoneo contenido, sin líquido libre en cavidad peritoneal (figs. 2-4). Fue indicada cirugía urgente y llevada a pabellón quirúrgico donde se realizó incisión media infraumbilical de piel y celular y se encontró una malla protésica antigua y, por debajo de ella, un saco herniario desplazado hacia el lado derecho con asas de intestino delgado. Al incidir la malla y con la apertura del saco se encontró líquido intestinal purulento con la serosa de las asas, con múltiples adherencias a este. Se tuvieron que liberar cuidadosamente y el peritoneo con fibrina, con un segmento de íleon acodado, presentó una perforación en el borde antimesentérico de $5 \mathrm{~mm}$ de diámetro, de bordes irregulares, producida por la punta afilada de un trozo de hueso de pollo inmediatamente antes de esta acodadura (figs. 5,6). Después de la extracción del cuerpo extraño se realizó un lavado peritoneal abundante, una resección de $23 \mathrm{~cm}$ de íleon con una anastomosis laterolateral con stappler lineal y posterior laparorrafia convencional, sin colocación de malla. Permaneció hospitalizada con tratamiento antibiótico y analgésico endovenoso, reposo digestivo al inicio y realimentación oral progresiva después. Reinterrogada dirigidamente, recordó haber ingerido un trozo de pollo asado hacía 3 meses, pero sin darse cuenta o haber tenido molestias en la deglución del trozo óseo. Se le dio el alta al sexto día en buenas condiciones. Se evaluó al mes y seguía en buenas condiciones. El resultado de la biopsia mostraba $23 \mathrm{~cm}$ de trozo de intestino delgado con su meso con perforación de $5 \mathrm{~mm}$ en borde antimesentérico y con su serosa inflamada con signos de peritonitis aguda. Al 7.0 mes reconsultó en el policlínico de cirugía por nueva recidiva herniaria (fig. 7), por lo que después de ser enviada a nutricionista para bajar de peso y completar estudio preoperatorio, se dejó en lista de espera para su hernioplastia definitiva. 


\section{DISCUSIÓN}

La ingestión de CE debió ocurrir desde el comienzo de la humanidad así como su complicación más temida, la perforación intestinal con una posterior peritonitis aguda, y pudo haber sido la causa de muerte de más de un «cólico miserere». Para dar una idea de la magnitud del problema, Rodríguez-Hermosa reportaba ${ }^{5}$ en el año 2009 que en los Estados Unidos 1500 personas morían anualmente por complicaciones por la ingesta de un $\mathrm{CE}$; en la antigüedad se desconocían este tipo de defunciones por esta causa. De ahí la importancia de contar con el primer reporte de Nonant ${ }^{12}$, ya indexado en la literatura médica occidental en el año 1841, en el que se informa que esta fue la causa de muerte de un paciente ingresado y tratado durante varios días sin éxito en su servicio y cuya etiología se desconocía posterior a su deceso. También podemos conocer que ya en ese entonces algunos médicos practicaban la autopsia clínica como parte de su deseo de llegar a un diagnóstico certero, siguiendo las enseñanzas de Rudolf Virchow y Carl von Rokitansky, y de su necesidad de reportarlas en revistas clínicas de su época, como un caso clínico de una etiología que hasta el día de hoy desafía y sorprende a los médicos de urgencia en su diagnóstico y tratamiento.

Al consultar la paciente en nuestro servicio de urgencia por dolor agudo e intenso localizado en relación a masa en región de la fosa iliaca derecha, con antecedentes de abdominoplastia antigua y de hernia recidivada hacía 3 años (según la clasificación de Chevrel, una M3W2R2) ${ }^{19}$ de la línea media subumbilical, con anillo herniario entre 5 a $10 \mathrm{~cm}$ y doblemente recidivada, el hecho de que no presentara signos de irritación peritoneal o de obstrucción intestinal planteaba un difícil diagnóstico ${ }^{10}$. La leucocitosis y la PCR elevadas orientaban a un inicio de un proceso infeccioso que podía estar relacionado con el dolor de su hernia incisional. La falta del dato anamnésico de la ingesta 3 meses antes de forma involuntaria de un trozo de hueso de pollo hizo más difícil este diagnóstico ${ }^{13}$, por lo que plantear la posibilidad de una hernia atascada complicada era lo más pertinente. El diagnóstico certero 
y específico del TAC corroboró lo reportado en la literatura ${ }^{10}$ al indicar que se trataba de una perforación de asa herniada por un cuerpo extraño con neumoperitoneo localizado lo que permitió llegar a un diagnóstico precoz. Mediante una laparotomía de urgencia encontramos que el asa intestinal herniada estaba por debajo de una malla preaponeurótica y sobre la pared aponeurótica, acodada con la perforación en su borde antimesentérico inmediatamente antes de la curva, lo que indicaba que el hueso permaneció ahí por varias semanas, equivaliendo este acodamiento a otro sitio de lentitud y riesgo de perforación por $\mathrm{CE}$, y según está descrito, esta complicación se puede demorar meses o años,9,10. La ingestión de CE fue facilitada porque la paciente era edentada parcial y usaba prótesis dental, por lo que tragó el CE sin percatarse y este fue horadando lentamente la pared del asa. Aunque en este caso ya se disponía del diagnóstico certero del TAC, se decide una inmediata laparotomía exploradora, ya que una laparoscopia carecía de sentido por estar la perforación dentro de un saco herniario. Una resección intestinal del segmento comprometido con anastomosis posterior fue lo adecuado ${ }^{1,10}$ y se sugiere incluso que cuando esta perforación compromete al colon en medio de una peritonitis aguda, debe realizarse una operación de Hartmann ${ }^{1}$.

Las perforaciones intestinales y sus severas complicaciones sépticas han estado presentes en la literatura médica desde hace mucho tiempo. Ya fueron publicadas por Nonat ${ }^{12}$ en 1841 y por Ginzburg ${ }^{20}$ en 1927. Kilbourne fue uno de los primeros en reportar un caso similar al nuestro en $1948^{21}$, es decir, de una perforación intestinal por hueso de pollo en asa herniada. Después, Giacom² ${ }^{22}$ en 1995, reportó dos casos: uno en una hernia inguinal y otro en una incisional que ambos debutaron con peritonitis aguda. Tay ${ }^{23}$ en el año 2013, describe uno en una hernia inguinal irreductible. Alharbi ${ }^{24}$ en 2016 describe otro en una hernia paraumbilical atascada. Cabe destacar especialmente el publicado por $\mathrm{Kilic}^{25}$, muy similar en su presentación y etiología al nuestro. Todos los casos mencionados coinciden en varios aspectos: que esta patología no representa fronteras, es poco frecuente, con 
una presentación diagnóstica que orienta en un principio a una apendicitis o peritonitis aguda, que el TAC cuando está disponible es de gran ayuda en el diagnóstico preoperatorio, que tiene una indicación quirúrgica urgente y que el acodamiento de las asas herniadas dentro del saco es el sitio donde la parte afilada del extremo del trozo de hueso de pollo se detiene y permanece hasta perforar después de un tiempo la pared intestinal. Giacom $^{22}$ comenta que el asa intestinal herniada, al estar acodada con su lumen distal disminuido por la comprensión extrínseca, debe soportar una mayor presión interna en su pared y cuando esta se ve debilitada, el cuerpo extraño punzante comienza a horadarla y, al aumentar la presión, este se rompe.

Creemos que la principal enseñanza que nos deja este caso, como personas y como médicos, válida para nosotros mismos, nuestras familias y pacientes, se relaciona con lo indicado por Moreno $\mathrm{Egea}^{1}$ en su publicación de 1996, donde destaca que las severas complicaciones por la ingestión de un CE se deben en parte a que la ingesta de los alimentos se hace de forma rápida y acelerada, sin dar tiempo a una óptima masticación y un manejo adecuado del bolo alimenticio antes de su deglución. Y junto a ello, que a diferencia de otros animales que sí tienen capacidad de tragar y digerir grandes porciones de alimentos y huesos sin masticarlos previamente (como por ejemplo leones, hienas, cocodrilos, jabalíes, etc.), nosotros tenemos un tubo digestivo no apto para recibir un CE sin riesgo de romperse, por lo que debemos cuidar aquello que ingerimos.

Por el hecho de tener una hernia con peritonitis aguda localizada, y ser del tipo 3 según los criterios de Losanoff ${ }^{26}$, correspondía realizar solo una herniorrafia sin uso de malla protésica por riesgo de infección de esta, y esto fue precisamente lo realizado. Dayton ${ }^{27}$ también reporta que cuando se usa la malla de polipropileno (Marlex), la única disponible en nuestro servicio de urgencia para reparar hernias de la pared abdominal, se observa una alta incidencia de infección crónica relacionada con la malla. Nuestra conducta es avalada por Gianom ${ }^{22}$, que sugiere que en estos casos la reparación herniaria 
debe realizarse 2 meses después de la resolución de la peritonitis. Además, el riesgo de fistulizarse la anastomosis era alto, por lo que creemos que fue adecuado diferir su hernioplastia definitiva.

En conclusión, debemos recordar la posibilidad de un cuerpo extraño como causa de obstrucción y abdomen agudo por perforación intestinal en cierto tipo de pacientes (ancianos, mala dentadura, etc.), para poder actuar con rapidez y evitar demoras innecesarias que puedan retrasar la cirugía y elevar la morbilidad. Un cuerpo extraño puede complicar la evolución de una recidiva ya conocida.

\section{BIBLIOGRAFÍA}

1. Moreno A, Aguayo J, Parrilla P. Perforaciones intestinales por cuerpos extraños (12 casos). Cir Esp. 1995;58:343-5.

2. Samuel DO, Adegboyega OF, Ene OM. Spontaneous expulsion of ingested foreign bodies: case series and review of literature. Am J Med Case Reports. 2015;3(9):272-5.

3. Acosta L, Cabañas S, Fleitas D, Chamorro C, Portillo P. Perforación colónica por cuerpo extraño. Rev. Nac. Itauguá. 2018;10(1):124-30.

4. Soria V, González P, Carrasco M. Perforación intestinal por cuerpo extraño tratada mediante laparoscopía. Gastroenterol Hepatol. 2000;23(4):211-12.

5. Rodríguez-Hermosa J, Cañete N, Artigau E, Girones J, Planellas P, CodinaCazador A. Perforación intestinal por un inusual cuerpo extraño. Rev Esp Enferm Dig. 2009;101(9):639-40.

6. Ramos R, Duarte P, Vicente C, Casteleiro C. Hemorragia digestiva alta desencadenada por un cuerpo extraño. Rev Esp Enferm Dig. 2008;100(4):238-9.

7. Sardiñas $P$, Hernández L, Pinza J. Perforación intestinal por espina de pescado: a propósito de un caso. Ccm. 2016;20(2):420-5.

8. Rodríguez-Hermosa J, Ruiz-Feliú B, Roig-García J, Albiol-Quer M, PlanellasGiné $\mathrm{P}$, Codina-Cazador $\mathrm{A}$. Lethal intestinal perforation after foreign body 
ingestion in a superobese patient. Obes Surg. 2009;19(8):1183-5. DOI: 10.1007/s11695-008-9667-8

9. Chan K. Perforation of Meckel's diverticulum caused by a chicken bone: A case report. J Med Case Rep. 2009;3(1):48. DOI: 10.1186/1752-1947-3-48

10. Barragán C, Rueda J, Espitia E, Uriza L, Rosselli D. Perforación intestinal por espina de pescado, revisión de la literatura científica y presentación de dos casos. Rev Colomb Cir. 2012;27(1):79-4.

11. Sierra-Solis A. Perforaciones intestinales por espinas de pescado: infrecuentes y curiosas. Semergen. 2013;39(2):117-8. DOI: 10.1016/j.semerg.2012.07.009

12. Monat M. Société Medicale d'Emulation: Phlebitis from a fish-bone, which passed through the stomach, and penetrated the mesenteric vein. Prov Med Surg J. 1841;3(56):77.

13. Joglekar S, Rajput I, Kamat S, Downey S. Sigmoid perforation caused by an ingested chicken bone presenting as right iliac fossa pain mimicking appendicitis: a case report. J Med Case Rep. 2009;3:7385. DOI: 10.4076/1752-1947-3-7385

14. Piñero A, Fernández J, Carrasco M, Riquelme J, Parrilla P. Intestinal perforation by foreign bodies. Eur J Surg. 2000;166(4):307-9. DOI: 10.1080/110241500750009140

15. Maglinte D, Taylor S, Ng A. Gastrointestinal perforation by chicken bones. Radiology. 1979;130(3):597-9. DOI: 10.1148/130.3.597

16. Vasapollo L, Chiarot M, Gallinaro L, Papaspyropoulos V, Montesano G, Ciulli $A$, et al. Perforazione intestinale da osso di pollo: a proposito di un caso clinic. Ann Ital Chir. 1997;68(4):555-8.

17. Rodríguez-Hermosa J, Farrés R, Codina A, Olivet F, Gironés J, Roig J, et al. Perforaciones intestinales causadas por cuerpos extraños. Cir. Esp. 2001;69(5):504-6.

18. Rodríguez-Hermosa J, Codina-Cazador A, Sirvent JM, Martìn A, Gironès J, Garsot E. Surgically treated perforations of the gastrointestinal tract caused 
by ingested foreign bodies. Colorrectal Dis. 2008;10(7):701-7. DOI: 10.1111/j.1463-1318.2007.01401.x

19. Chevrel J, Rath A. Classification of the incisional hernias of the abdominal wall. Hernia 2000;4(1):7-11. DOI: 10.1007/BF01230581.

20. Ginzburg L, Beller A. The clinical manifestations of non-metallic perforating intestinal foreign bodies. Ann Surg. 1927;86(6):928-39. DOI: 10.1097/00000658-192712000-00016

21. Kilbourne B. Perforation of Ileum by Ingested Chicken Bone Complicating Ventral Hernia. Ann Surg. 1948;127(6):1226-31. DOI: 10.1097/00000658194806000-00012

22. Gianom D, Fenner A. Dünndarmperforation bei Hernienträgern [Perforation of the small - intestine in patients with hernia]. Chirurg. 1995;66(6):637-9.

23. Tay G, Chng J, Wong W, Goh Y. Chicken bone perforation of an irreducible inguinal hernia: a case report and review of the literature. Hernia. 2013;17(6):805-7. DOI: 10.1007/s10029-012-0980-8

24. Alharbi M. Foreign Body (Chicken Bone) Leading to Perforation of the Small Bowel within Irreducible Paraumbilical Hernia: Case Report. J Surg Open Access. 2016;2(4):2-125. DOI: 10.16966/2470-0991.125

25. Kilic E, Ata S, Kly C, Simsek T, Zafer N. Perforation due to foreign body in incarcerated incisional hernia. Causa Pedia. 202;9(1):24-7.

26. Losanoff J, Basson M. Amyand hernia: A classification to improve management. Hernia. 2008;12(3):325-6. DOI: 10.1007/s10029-008-0331-y

27. Dayton M, Buchele B, Shirazi S, Hunt L. Use of an absorbable mesh to repair contaminated abdominal-wall defects. Arch Surg. 1986;121(8):954-60. DOI: 10.1001/archsurg.1986.01400080100019 


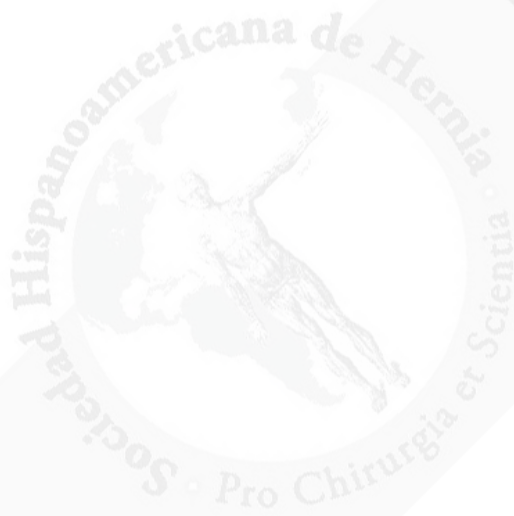




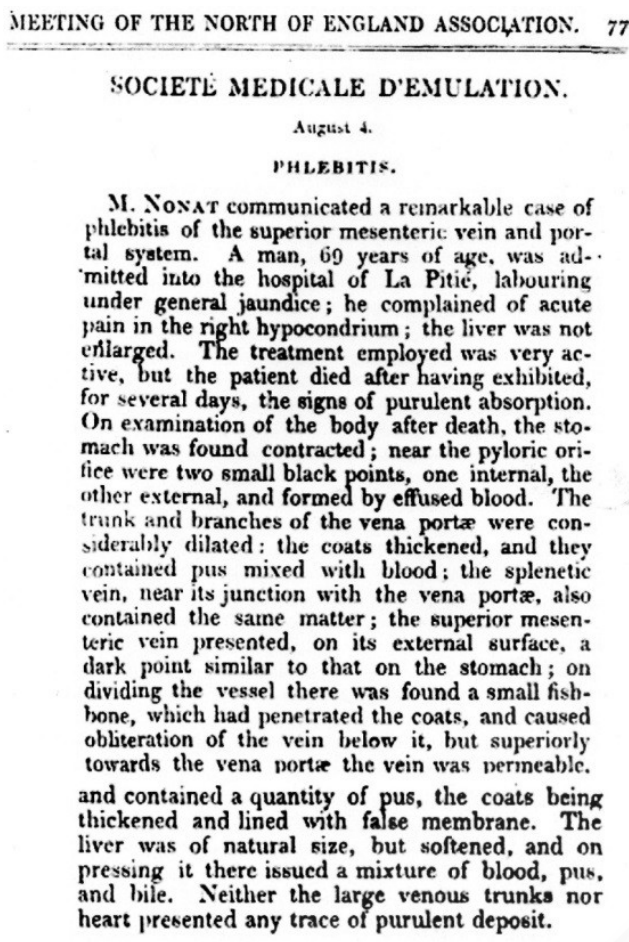

Fig. 1. Primer reporte mundial indexado el año 1841 en los primeros números del Provincial Medical and Surgical Journal (actualmente British Medical Journal) de M. Nonat sobre un caso clínico por perforación intestinal por espina de pescado. Se informa de un paciente que ingresó a su hospital con un abdomen agudo y síndrome ictérico que evolucionó con septicemia. La autopsia mostró en región abdominal superior una perforación prepilórica transfixiante con una espina de pescado que también había perforado la pared lateral de la vena mesentérica superior, causando un hemoperitoneo localizado y una pileflebitis aguda. Nota: antiguamente en Francia, a los escasos y conocidos médicos e investigadores, se les citaba en la literatura médica anteponiéndoles la letra M. (de monsier) a los hombres y M.M (de madame) a las mujeres. Además, en este caso, se especificaba que era del Hospital La Pitié-Salpêtrière (La Piedad de Sal de Piedra) para diferenciarlo de su hermano $\mathrm{M}$. Nonat que trabajaba en el vecino hospital parisino de La Charité. 


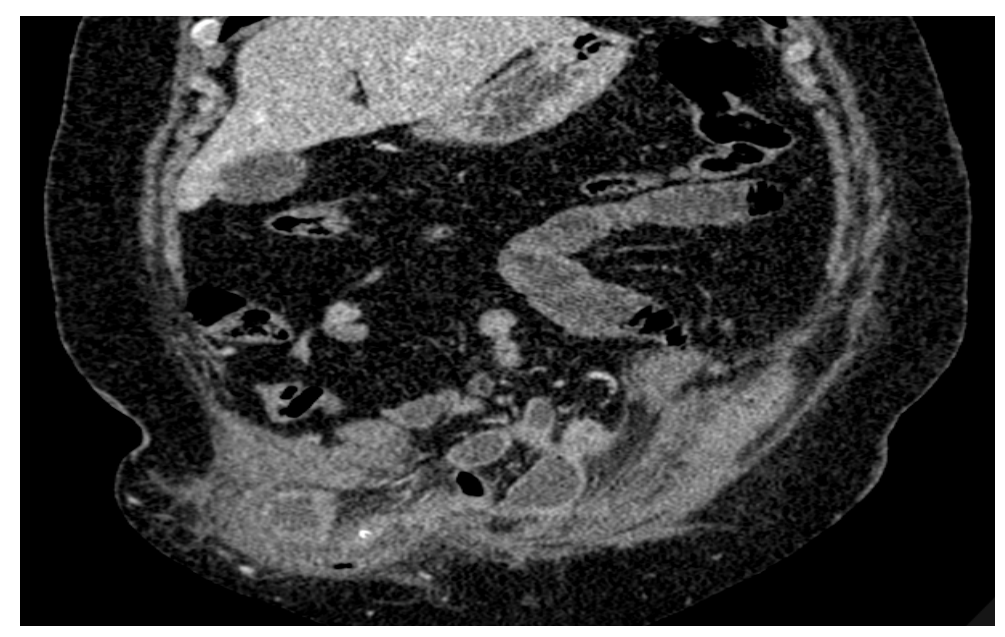

Fig. 2. Corte coronal de TAC de abdomen y pelvis que en región inferior derecha muestra asa intestinal herniada con punto blanquecino en medio de un asa. Se observan también partes de asas intraabdominales dilatadas. 


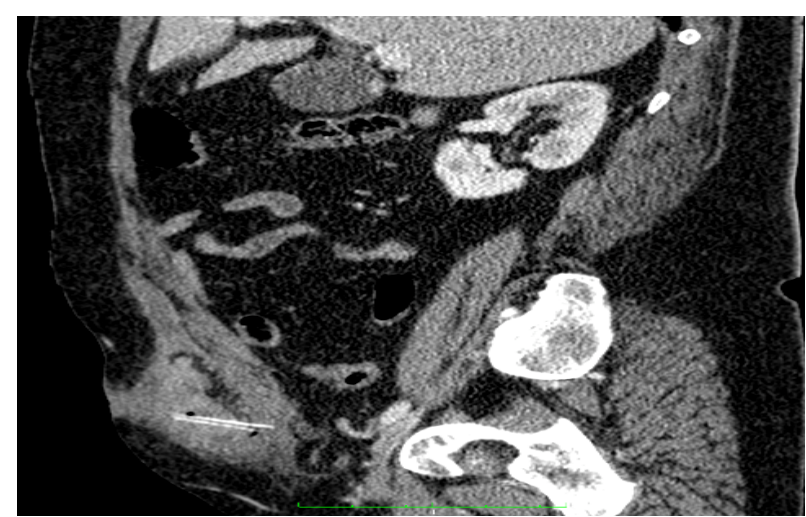

Fig. 3. Corte sagital de TAC de abdomen y pelvis, que muestra en asa herniada trozo blanquecino de cuerpo extraño con un lumen en medio de asa herniada.

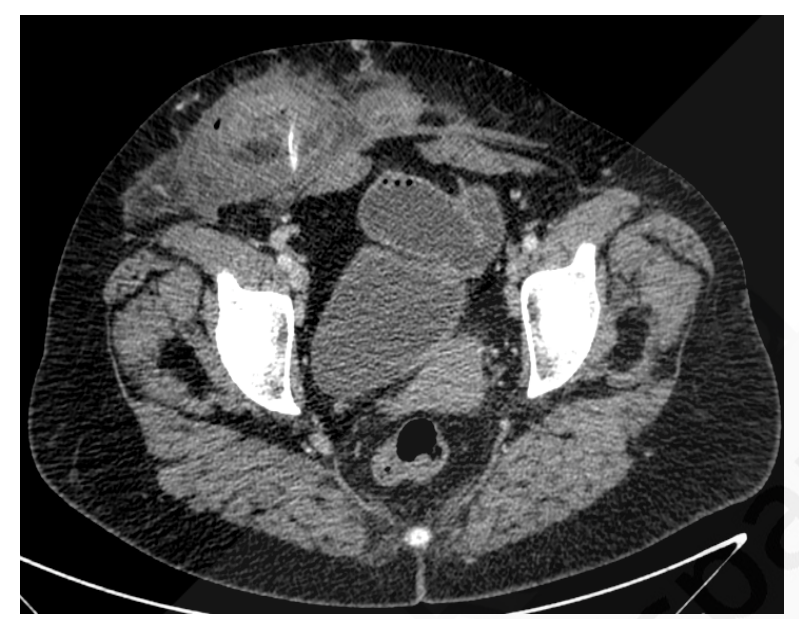

Fig. 4. Corte axial de TAC que muestra asa herniada con cuerpo extraño en su interior. 


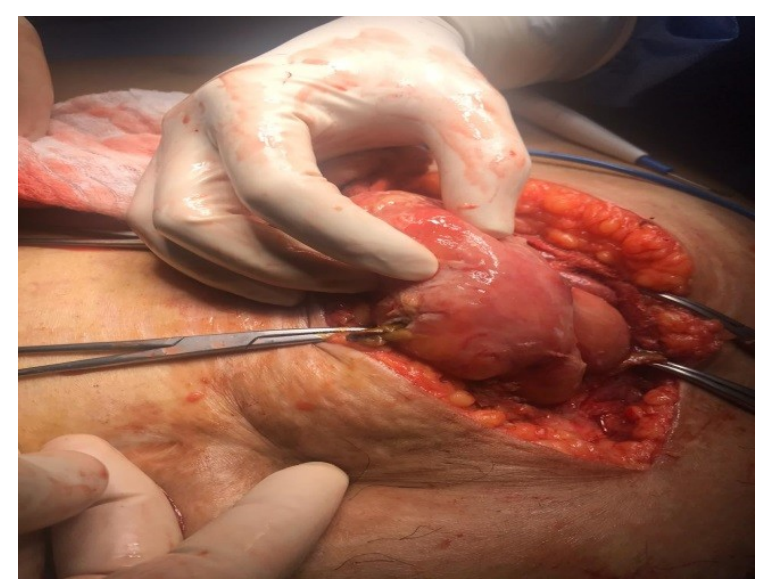

Fig. 5. Foto del intraoperatorio que muestra incisión de piel y celular con saco herniario abierto y en parte inferior de borde antimesentérico de asa herniada una perforación de un cuerpo extraño puntiagudo que comienza a ser traccionado por pinza Kelly.

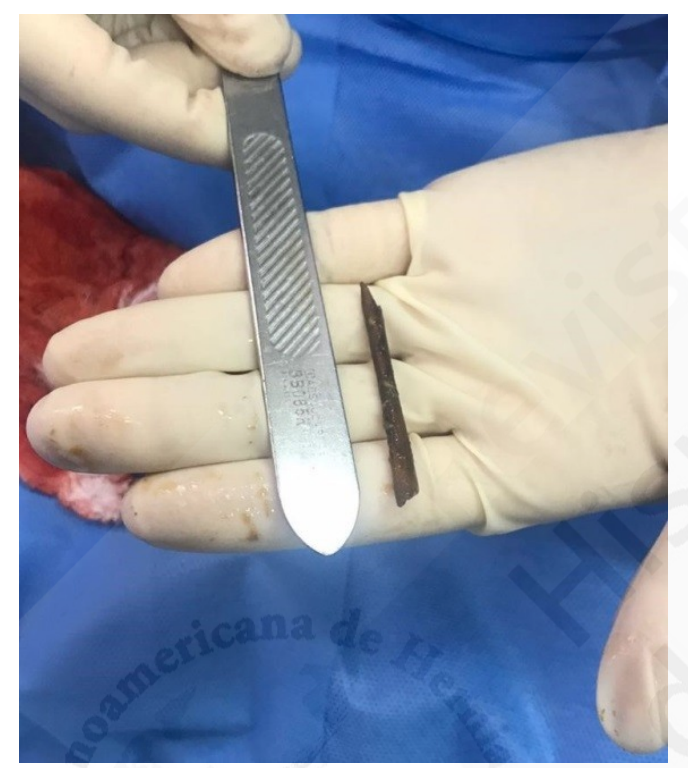

Fig. 6. Foto de cuerpo extraño extraído que corresponde a un trozo de hueso de pollo rostizado y con una punta muy afilada de unos $4.5 \mathrm{~cm}$ de largo. 


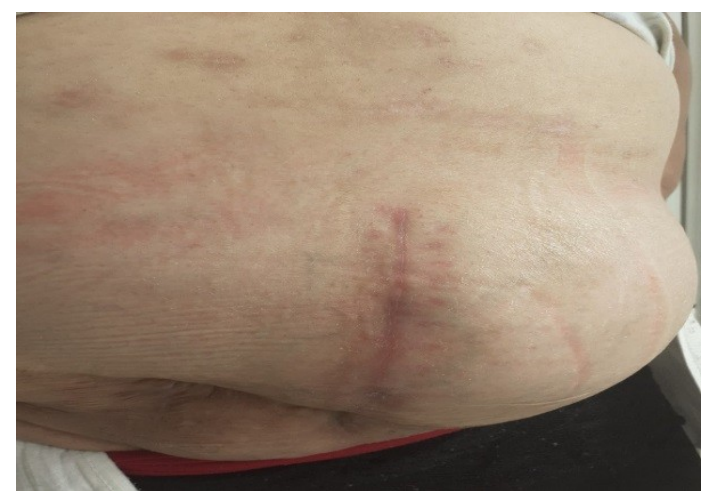

Fig. 7. Foto de abdomen de la paciente después de 5 meses de la cirugía, con cicatriz retráctil en región inferior y ausencia de ombligo por antigua abdominoplastia y cicatriz de LMIU reciente con recidiva herniaria. 